\title{
A comparative analysis of two methods for the calculation of electric-field-induced perturbations to molecular vibration
}

\author{
Josep Martí ${ }^{\text {a) }}$ and David M. Bishop \\ Department of Chemistry, University of Ottawa, Ottawa, Canada K1N 6 N5
}

(Received 20 April 1993; accepted 7 May 1993)

\begin{abstract}
Two common methods of accounting for electric-field-induced perturbations to molecular vibration are analyzed and compared. The first method is based on a perturbation-theoretic treatment and the second on a finite-field treatment. The relationship between the two, which is not immediately apparent, is made by developing an algebraic formalism for the latter. Some of the higher-order terms in this development are documented here for the first time. As well as considering vibrational dipole polarizabilities and hyperpolarizabilities, we also make mention of the vibrational Stark effect.
\end{abstract}

\section{INTRODUCTION}

There is growing interest in the effect of electric fields on molecular vibration and this complements the picture we already have of the effects of an electric field on electronic motion. The latter effects are conventionally described in terms of the dipole polarizability $(\alpha)$ and hyperpolarizabilities $(\beta, \gamma, \quad$ etc. $){ }^{1}$ The vibrational counterparts of these intrinsic molecular properties are the main concern of this article. The level of the discussion will hopefully fill the gap between two recent reviews on the same subject, one detailed and specialist ${ }^{2}$ and the other of a more introductory and general character. ${ }^{3}$ Our principle objective is to clarify the relationships, not altogether transparent, which exist between two common methods for calculating electric-field-induced vibrational perturbations. Broadly speaking, we can categorize these methods as perturbation-theoretic (method A) and finite-field-based (method B). To make the comparison will require that we put method B on an algebraic footing. As well as considering vibrational polarizabilities and hyperpolarizabilities, we will also look at the vibrational Stark effect., ${ }^{4,5}$

For the sake of clarity and because it is sufficient to get our principal points across, we will make our analysis for a diatomic molecule, even though both methods can be used for polyatomic molecules. We will also only consider a static uniform electric field since method B is not capable of dealing with dynamic (oscillating) fields. This field will be applied along the nuclear axis $(z)$ and we will omit all subscripts, that is to say $\mu, \alpha$, etc., will imply the dipole moment and polarizability components $\mu_{z}, \alpha_{z z}$, etc. Other components are clearly accessible but are not necessary for the arguments which we wish to make. That we keep the electric field parallel to the nuclear axis implies that we are ignoring rotation or, in other words, our results are molecular-axis based rather than laboratory-axis based. The theory of relating the results found in these two axis systems has been discussed in detail in Ref. 2 .

It is pertinent to briefly refer to previous work which has been based on the two aforementioned methods. The

\footnotetext{
${ }^{2)}$ Permanent address: Institute for Computational Chemistry and Department of Chemistry, Universitat de Girona, 17071 Girona, Spain.
}

perturbation-theoretic formulas for the dynamic vibrational polarizabilities and hyperpolarizabilities of polyatomic molecules have been given by Bishop and Kirtman ${ }^{6-8}$ and applied to $\mathrm{FH}, \mathrm{CO}_{2}, \mathrm{H}_{2} \mathrm{O}$, and $\mathrm{NH}_{3}{ }^{7,9}$ The same philosophy has been applied to changes to infrared spectra caused by an electric field (the vibrational Stark effect) with $\mathrm{CO}$ as an example. ${ }^{4}$ The finite field approach to vibrational polarizabilities and hyperpolarizabilities was first introduced by Bishop and Solunac ${ }^{10}$ for the case of $\mathrm{H}_{2}^{+}$ and by Adamowicz and Bartlett ${ }^{11}$ for FH. The vibrational Schrödinger equation is solved in the presence of a small finite, static, uniform, electric field. The vibrational energy levels thereby become field dependent and, for any given level, a fit may be made to a Taylor series in the field and the coefficients related to the total (electronic and vibrational) polarizabilities. Subtraction of the known electronic values gives the vibrational contribution to a particular polarizability or hyperpolarizability. Duran and coworkers ${ }^{12}$ have used a variation of this method; they obtain potential curves in the presence of various finite electric fields, but rather than solve numerically (as Adamowicz and Bartlett did) for the vibrational energy levels, they obtain the curvature analytically (at the field-perturbed equilibrium geometry) for the various fields $(F)$. From this, the field-perturbed harmonic vibrational frequency, $\omega_{e}(F)$, is found. The energy for the vibrational level with vibrational quantum number $n$ is then $V\left(Q_{e}\right)+(n$ $\left.+\frac{1}{2}\right) \hbar \omega_{e}(F)$, where $Q_{e}$ is the field-dependent equilibrium geometry. Numerical differentiation with respect to $F$ gives two components to the vibrational polarizability and hyperpolarizabilities; one is related to the shift in the equilibrium geometry, i.e., to $Q_{e}(F)$, and which they called "nuclear relaxation" and the other is related to $\omega_{e}(F)$ and was called simply "vibrational." As well as applying this methodology to vibrational polarizabilities and hyperpolarizabilities, ${ }^{12}$ they have also used it for calculating the vibrational Stark shifts for several molecules. ${ }^{5,13}$

In the next section both methods will be described and analyzed in more detail and in the context of a diatomic molecule vibrating in its lowest vibrational state. Certain higher-order perturbation-theoretic contributions to the vibrational (hyper)polarizabilities will be given for the first 
time. An extension to vibrationally excited states is given in Sec. III and the trivial relation of the foregoing results to the vibrational Stark effect is to be found in Sec. IV.

\section{ANALYSIS: THE VIBRATIONAL GROUND STATE OF A DIATOMIC MOLECULE}

\section{A. Method A}

For a diatomic molecule vibrating in a static uniform electric field $(F)$ acting along its axis, the potential curve includes the perturbation

$$
H^{\prime}=-\mu F-\frac{1}{2} \alpha F^{2}-\frac{1}{6} \beta F^{3}-\cdots,
$$

where $\mu, \alpha, \beta, \ldots$ are the dipole moment, dipole polarizability, and first hyperpolarizability functions, respectively. From perturbation theory the first-order correction to the ground state vibrational energy will therefore include terms of the type $\left\langle\psi_{0}|\xi| \psi_{0}\right\rangle$, with $\xi=\mu, \alpha, \beta, \ldots$, where $\psi_{0}$ is the unperturbed ground state vibrational wave function. The difference between $\left\langle\psi_{0}|\xi| \psi_{0}\right\rangle$ and $\xi^{0}=\xi\left(Q_{e}\right)$, the electronic value of the property $\xi$ at the unperturbed equilibrium geometry $\left(Q_{e}\right)$, is called the zero-point-vibrationalaveraging correction $\xi^{\mathrm{ZP}}$, i.e.,

$$
\left\langle\psi_{0}|\xi| \psi_{0}\right\rangle=\xi^{0}+\xi^{Z P} \text {. }
$$

We may express any electric property $\xi$ as a truncated Taylor series by

$$
\xi=\xi^{0}+(\partial \xi / \partial Q) Q+\frac{1}{2}\left(\partial^{2} \xi / \partial Q^{2}\right) Q^{2}
$$

(higher-order derivatives being ignored). In Eq. (3) $Q$ is the normal coordinate $m^{1 / 2}\left(R-R_{e}\right)$, where $m$ is the reduced nuclear mass and $R$ is the internuclear separation. The second and third terms in Eq. (3) are referred to as the electrical harmonicity and anharmonicity, respectively. The wave function $\psi_{0}$ can be found as a perturbed harmonic oscillator wave function, the perturbation (mechanical anharmonicity) being expressed by the cubic force constant $k^{\prime}$. That is, we can write the potential curve (with no electric field present) in the form

$$
V=V^{0}+\frac{1}{2} \omega_{e}^{2} Q^{2}+\frac{1}{6} k^{\prime} Q^{3},
$$

where $\nu^{0}$ is the potential at $Q_{e}$ and $\omega_{e}$ is the circular harmonic vibrational frequency. Combining Eqs. (2)-(4) and using standard values for the integrals over harmonic oscillator functions ${ }^{14}$ leads to the well-known formula

$$
\xi^{\mathrm{ZP}}=\left(\hbar / 4 \omega_{e}\right)\left[\left(\partial^{2} \xi / \partial Q^{2}\right)-\omega_{e}^{-2} k^{\prime}(\partial \xi / \partial Q)\right] .
$$

This vibrational correction is quite independent of any effect the electric field has on the vibrational motion. In the double harmonic approximation, where both $\partial^{2} \xi / \partial Q^{2}$ and $k^{\prime}$ are ignored, the zero-point-vibrational correction for any electric property will be zero.

Higher-order perturbation-theoretic corrections to the ground state vibrational energy due to the electric field will lead to further corrections to the polarizability $\alpha$ and first hyperpolarizability $\beta$. The vibrational polarizabilities can be written in the form ${ }^{2,7,8}$
TABLE I. Formulas for the different contributions to $\alpha^{v}$ and $\beta^{v}$.

\begin{tabular}{ll}
\hline \hline Term & \multicolumn{1}{c}{ Formula } \\
\hline$\left[\mu^{2}\right]^{0,0}$ & $\omega_{e}^{-2}(\partial \mu / \partial Q)^{2}$ \\
{$\left[\mu^{2}\right]^{2,0}$} & $(1 / 8) \hbar \omega_{e}^{-3}\left(\partial^{2} \mu / \partial Q^{2}\right)^{2}$ \\
{$\left[\mu^{2}\right]^{1,1}$} & $-(3 / 4) \hbar \omega_{e}^{-5}\left(k^{\prime}\right)(\partial \mu / \partial Q)\left(\partial^{2} \mu / \partial Q^{2}\right)$ \\
{$\left[\mu^{2}\right]^{0,2}$} & $(3 / 8) \hbar \omega_{e}^{-7}\left(k^{\prime}\right)^{2}(\partial \mu / \partial Q)^{2}$ \\
{$[\mu \alpha]^{0,0}$} & $(3) \omega_{e}^{-2}(\partial \mu / \partial Q)(\partial \alpha / \partial Q)$ \\
{$[\mu \alpha]^{2,0}$} & $(3 / 8) \hbar \omega_{e}^{-3}\left(\partial^{2} \mu / \partial Q^{2}\right)\left(\partial^{2} \alpha / \partial Q^{2}\right)$ \\
{$[\mu \alpha]^{1,1}$} & $-(9 / 8) \hbar \omega_{e}^{-5}\left(k^{\prime}\right)\left[(\partial \mu / \partial Q)\left(\partial^{2} \alpha / \partial Q^{2}\right)+(\partial \alpha / \partial Q)\right.$ \\
& $\left.\times\left(\partial^{2} \mu / \partial Q^{2}\right)\right]$ \\
{$[\mu \alpha]^{0,2}$} & $(9 / 8) \hbar \omega_{e}^{-7}\left(k^{\prime}\right)^{2}(\partial \mu / \partial Q)(\partial \alpha / \partial Q)$ \\
{$\left[\mu^{3}\right]^{1,0}$} & $(3) \omega_{e}^{-4}(\partial \mu / \partial Q)^{2}\left(\partial^{2} \mu / \partial Q^{2}\right)$ \\
{$\left[\mu^{3}\right]^{0,1}$} & $-(1) \omega_{e}^{-6}\left(k^{\prime}\right)(\partial \mu / \partial Q)^{3}$ \\
{$\left[\mu^{3}\right]^{2,1}$} & $-(45 / 16) \hbar \omega_{e}^{-7}\left(k^{\prime}\right)(\partial \mu / \partial Q)\left(\partial^{2} \mu / \partial Q^{2}\right)^{2}$ \\
{$\left[\mu^{3}\right]^{1,2}$} & $(63 / 16) \hbar \omega_{e}^{-9}\left(k^{\prime}\right)^{2}(\partial \mu / \partial Q)^{2}\left(\partial^{2} \mu / \partial Q^{2}\right)$ \\
{$\left[\mu^{3}\right]^{0,3}$} & $-(21 / 16) \hbar \omega_{e}^{-11}\left(k^{\prime}\right)^{3}(\partial \mu / \partial Q)^{3}$ \\
{$\left[\mu^{3}\right]^{3,0}$} & $(3 / 16) \hbar \omega_{e}^{-5}\left(\partial^{2} \mu / \partial Q^{2}\right)^{3}$ \\
\hline \hline
\end{tabular}

${ }^{a}$ The last four formulas are deduced from method B.

$$
\begin{aligned}
\alpha^{\nu}= & 2 \hbar^{-1} \sum_{k}^{\prime} \omega_{k}^{-1}\langle 0|\mu| k\rangle\langle k|\mu| 0\rangle=\left[\mu^{2}\right], \\
\beta^{v}= & 6 \hbar^{-2} \sum_{k}^{\prime} \sum_{l}^{\prime} \omega_{k}^{-1} \omega_{l}^{-1}\langle 0|\mu| k\rangle\langle k|\bar{\mu}| l\rangle\langle l|\mu| 0\rangle \\
& +6 \hbar^{-1} \sum_{k}^{\prime} \omega_{k}^{-1}\langle 0|\mu| k\rangle\langle k|\alpha| 0\rangle \\
= & {\left[\mu^{3}\right]+[\mu \alpha], }
\end{aligned}
$$

where the primes on the summations indicate exclusion of the ground state, $\omega_{k}$ is the circular transition frequency for the $k$ th fundamental level, and $|k\rangle$ is the corresponding wave function, and $\bar{\mu}$ in Eq. (7) is $\mu-\langle 0|\mu| 0\rangle$. Using Eqs. (3) and (4), $\left[\mu^{2}\right],\left[\mu^{3}\right]$, and $[\mu \alpha]$ may be evaluated to various orders ${ }^{7,8}$ in electrical and mechanical anharmonicity. A particular order is denoted by $[\ldots]^{n, m}$, where $n$ indicates that a second derivative of an electric property with respect to $Q$ occurs $n$ times and $m$ indicates that $k^{\prime}$ occurs $m$ times. In Table I we give expressions for $[. . .]^{n, m}$ such that $\alpha^{v}$ and $\beta^{v}$ can be evaluated to the following overall order:

$$
\begin{aligned}
\alpha^{v}= & {\left[\mu^{2}\right]^{0,0}+\left[\mu^{2}\right]^{2,0}+\left[\mu^{2}\right]^{1,1}+\left[\mu^{2}\right]^{0,2} } \\
\beta^{v}= & {\left[\mu^{3}\right]^{1,0}+\left[\mu^{3}\right]^{0,1}+\left[\mu^{3}\right]^{0,3}+\left[\mu^{3}\right]^{1,2}+\left[\mu^{3}\right]^{2,1} } \\
& +\left[\mu^{3}\right]^{3,0}+[\mu \alpha]^{0,0}+[\mu \alpha]^{2,0}+[\mu \alpha]^{1,1}+[\mu \alpha]^{0,2} .
\end{aligned}
$$

Certain combinations of anharmonicities, e.g., $\left[\mu^{2}\right]^{1,0}$, are excluded on the grounds of symmetry. Details for the construction of the formulas in Table I can be found in Refs. 7 and 8. It should be noted that when $m \geqslant 2$, account must be taken of the mechanical-anharmonic effects in the energies in the denominators in Eqs. (6) and (7) and that the vibrational wave functions must be developed to second order or higher in $k^{\prime}$.

The final, total, vibrational contribution to $\alpha$ and $\beta$ may then be defined by

$$
\alpha(\mathrm{vib})=\alpha^{\mathrm{ZP}}+\alpha^{v}
$$




$$
\beta(\mathrm{vib})=\beta^{\mathrm{ZP}}+\beta^{v},
$$

and the energy of the lowest vibrational state in the presence of the electric field $(F)$ is

$$
\begin{aligned}
E= & E^{0}-\left(\mu^{0}+\mu^{\mathrm{ZP}}\right) F-\frac{1}{2}\left[\alpha^{0}+\alpha(\mathrm{vib})\right] F^{2} \\
& -\frac{1}{6}\left[\beta^{0}+\beta(\mathrm{vib})\right] F^{3}-\cdots .
\end{aligned}
$$

\section{B. Method B}

To make the desired connection between method $\mathrm{A}$ and the finite-field method it is necessary to put the latter on an algebraic basis. This can be done by expressing the potential curve of the diatomic molecule in the presence of the field $(F)$ as a power series expansion

$$
V(Q, F)=\sum_{n} \sum_{m} a_{n m} Q^{n} F^{m}
$$

There are obvious identities between the coefficients $a_{n m}$ in Eq. (12) and the derivatives used in method A, e.g., $a_{11}$ $=-(\partial \mu / \partial Q), \quad a_{12}=-\frac{1}{2}(\partial \alpha / \partial Q), \quad a_{22}=-\frac{1}{4}\left(\partial^{2} \alpha / \partial Q^{2}\right)$. From our point of view, the field-perturbed curve differs in two respects from the unperturbed one (a) the equilibrium geometry (position of minimum energy) is displaced, and (b) the curvature at the field-perturbed minimum is altered. Both these changes will affect the vibrational energies and thereby introduce two types of vibrational polarizability (hyperpolarizability) which we will label $\alpha$ (disp), $\beta$ (disp), and $\alpha$ (curv), $\beta$ (curv). We will first consider $\alpha$ (disp) and $\alpha$ (curv).

To discover the displacement term $\alpha$ (disp), called "nuclear relaxation" by Martí et al., ${ }^{12}$ it is necessary to differentiate $V(Q, F)$ with respect to $Q$ and set the result equal to zero. Solution of the resulting equation leads to a field-dependent equilibrium geometry given by

$$
\begin{aligned}
Q_{e}(F)= & -\left(2 a_{20}\right)^{-1}\left[a_{11} F+\left(a_{12}+3 a_{30} a_{11}^{2} / 4 a_{20}^{2}\right.\right. \\
& \left.\left.-a_{11} a_{21} / a_{20}\right) F^{2}+\cdots\right] .
\end{aligned}
$$

Inserting this formula into $V(Q, F)$, we arrive at

$$
V\left(Q_{e}, F\right)=a_{00}+a_{01} F+\left(a_{02}-a_{11}^{2} / 4 a_{20}\right) F^{2}+\cdots
$$

and we identify $\alpha$ (disp) by

$$
\alpha(\text { disp })=a_{11}^{2} / 2 a_{20}=\left[\mu^{2}\right]^{0,0} .
$$

The change in curvature affects the zero-point vibrational energy which, since we ignore third derivatives of electric properties, we can take as $\frac{1}{2} \hbar \omega_{e}(F)$. Where $\omega_{e}(F)$ is the field perturbed harmonic frequency and is related to the curvature by the equations

$$
\begin{aligned}
& \omega_{e}(F)=[k(F)]^{1 / 2}, \\
& k(F)=\left[\partial^{2} V(Q, F) / \partial Q^{2}\right]_{Q_{e}(F)} .
\end{aligned}
$$

Combining Eqs. (12), (17), and (13) we obtain

$$
\begin{aligned}
k(F)= & 2 a_{20}+\left(2 a_{21}-3 a_{30} a_{11} / a_{20}\right) F \\
& +\left[2 a_{22}-3 a_{30}\left(a_{12}+3 a_{30} a_{11}^{2} / 4 a_{20}^{2}\right.\right. \\
& \left.\left.-a_{11} a_{21} / a_{20}\right) / a_{20}\right] F^{2}+\cdots
\end{aligned}
$$

and using Eq. (16) and abstracting the $F^{2}$ term in $\frac{1}{2} \hbar \omega_{e}(F)$, we deduce that

$$
\begin{aligned}
\alpha(\text { curv })= & -\hbar \omega\left(2 a_{22} a_{20}-3 a_{30} a_{12}\right) / 4 a_{20}^{2}+\hbar \omega\left(a_{21}^{2} a_{20}^{2}\right. \\
& \left.-36 a_{30} a_{11} a_{21} a_{20}+27 a_{30}^{2} a_{11}^{2}\right) / 32 a_{20}^{4} .
\end{aligned}
$$

Or, in the language of method $\mathrm{A}$,

$$
\alpha(\text { curv })=\alpha^{\mathrm{ZP}}+\left[\mu^{2}\right]^{2,0}+\left[\mu^{2}\right]^{1,1}+\left[\mu^{2}\right]^{0,2} .
$$

The same procedure, but with the terms in $F^{3}$ consistently retained, gives

$$
\beta(\text { disp })=[\mu \alpha]^{0,0}+\left[\mu^{3}\right]^{1,0}+\left[\mu^{3}\right]^{0,1}
$$

and

$$
\begin{aligned}
\beta(\text { curv })= & \beta^{\mathrm{ZP}}+[\mu \alpha]^{2,0}+[\mu \alpha]^{1,1}+[\mu \alpha]^{0,2}+\left[\mu^{3}\right]^{3,0} \\
& +\left[\mu^{3}\right]^{2,1}+\left[\mu^{3}\right]^{1,2}+\left[\mu^{3}\right]^{0,3}
\end{aligned}
$$

Equations (15), (20)-(22), and

$$
\begin{aligned}
& \alpha(\text { vib })=\alpha(\text { disp })+\alpha(\text { curv }), \\
& \beta(\text { vib })=\beta(\text { disp })+\beta \text { (curv) }
\end{aligned}
$$

constitute the algebraic foundation of the finite field method.

\section{Comparison of the two methods}

With the results of the two previous subsections, we are now in a position to identify the connections between the two methods of calculation. The total vibrational contribution to the dipole polarizability can be written, Eqs. (10a) and (23), as

$$
\alpha(\text { vib })=\alpha^{\mathrm{ZP}}+\alpha^{\nu}=\alpha(\text { disp })+\alpha(\text { curv }) .
$$

However, the two contributions in each case are quite different; only if we know the value of $\left[\mu^{2}\right]^{0,0}$ can we compare the contributing parts, that is,

$$
\alpha(\operatorname{disp})=\left[\mu^{2}\right]^{0,0}
$$

and

$$
\alpha(\text { curv })=\alpha^{\mathrm{ZP}}+\alpha^{v}-\alpha(\text { disp }) .
$$

For the vibrational contribution to the first hyperpolarizability, the situation is the same. We can easily compare the total contributions through Eqs. (10b) and (24),

$$
\beta(\text { vib })=\beta^{\mathrm{ZP}}+\beta^{v}=\beta(\text { disp })+\beta(\text { curv }),
$$

but only if we know the sum of $[\mu \alpha]^{0,0},\left[\mu^{3}\right]^{1,0}$, and $\left[\mu^{3}\right]^{0,1}$ can we go further, and use

$$
\beta(\operatorname{disp})=[\mu \alpha]^{0,0}+\left[\mu^{3}\right]^{1,0}+\left[\mu^{3}\right]^{0,1}
$$

and

$$
\beta(\text { curv })=\beta^{\mathrm{ZP}}+\beta^{v}-\beta(\text { disp }) .
$$

So far in our comparison we have been concentrating on the two-step finite-field procedure of Martí et al. ${ }^{12}$ Turning now to Adamowicz and Bartlett's ${ }^{11}$ one-step pro- 
cedure, it is clear that it is only their total $\alpha$ (vib) and $\beta(\mathrm{vib})$ that can be compared with the $\left(\alpha^{\mathrm{ZP}}+\alpha^{\nu}\right)$ and $\left(\beta^{\mathrm{ZP}}\right.$ $+\beta^{v}$ ) of method $A$.

An obvious conclusion to be drawn from the foregoing is that, when method A is used, documentation of the $[\ldots]^{n, m}$ terms will be useful for a detailed comparison with any results from method $B$.

A frequently used approximation, known as the "double harmonic approximation," is to ignore all anharmonicities, mechanical and electrical, i.e., second derivatives (and higher) of the electric properties and $k^{\prime}$; this means $\alpha(\mathrm{vib}) \cong\left[\mu^{2}\right]^{0,0}=\alpha($ disp $)$ and $\beta($ vib $) \cong[\mu \alpha]^{0,0}$ and the latter cannot be equated to $\beta$ (disp). The same approximation implies, see Eq. (5), that there is no zero-point-vibrational averaging term.

\section{VIBRATIONAL EXCITED STATES}

We can now look at the vibrational polarizabilities and hyperpolarizabilities for a vibrationally excited state with vibrational quantum $v=n(n \neq 0)$. Within the context of method A, the zero-point-vibrational averaging is easily found from the well-known expression

$$
\xi^{\mathrm{ZP}}=\left(n+\frac{1}{2}\right)\left(\hbar / 2 \omega_{e}\right)\left[\left(\partial^{2} \xi / \partial Q^{2}\right)-\omega_{e}^{-2} k^{\prime}(\partial \xi / \partial Q)\right]
$$

but the formulas for $\alpha^{v}$ and $\beta^{v}$ for $n \neq 0$ are another matter altogether. They have not yet been determined via method $\mathrm{A}$ and to do so would require much tedious algebra. However, for a diatomic molecule and a static field, method B can be extended to vibrationally excited states in an absolutely straightforward way, since the polarizabilities are based on the vibrational energies being $\left(n+\frac{1}{2}\right) \hbar \omega_{e}(F)$. Very simply we have

$$
\alpha(\text { vib })=\alpha(\text { disp })+(2 n+1) \alpha(\text { curv })
$$

and

$$
\beta(\text { vib })=\beta(\text { disp })+(2 n+1) \beta \text { (curv). }
$$

The displacement term is, of course, common to all levels. With respect to method A, it is both clear and logical that all terms in Table I containing the Dirac constant $(\hbar)$ will be multiplied by $(2 n+1)$ for a vibrationally excited state.

\section{VIBRATIONAL STARK EFFECT}

An important application of the theory which we have been discussing is with regard to the vibrational Stark effect, that is the effect of an electric field on a molecule's infrared spectrum (fundamental vibrational frequencies and intensities). For it is here that there is experimental evidence with which to compare the theory, see, e.g., Ref. 15. What we require, theoretically, is the shift $(\Delta \omega)$ in the frequency associated with the transition between the vibrational ground state and the fundamental state. For a diatomic molecule, ignoring any anharmonicity in the per- turbed potential curve (i.e., third and higher derivatives with respect to $Q$ of the electric properties), this shift will be

$$
\Delta \omega=-\hbar^{-1}\left[2 \mu^{\mathrm{ZP}} F+\alpha(\text { curv }) F^{2}+\frac{1}{3} \beta(\text { curv }) F^{3}+\cdots\right] .
$$

This equation comes from using the perturbation $H^{\prime}$ in Eq. (1), and Eqs. (32) and (33), and realizing that (a) the displacement terms are the same for both the ground and fundamental states, and (b) for the dipole moment function $\mu$ (curv) $=\mu^{\mathrm{ZP}}$, see Eq. (5). In a previous publication ${ }^{4}$ one of us used this formula, but ignored all but the "ZP" terms in $\alpha$ (curv). Equation (34) may, in principle, be compared with the work of Duran and co-workers ${ }^{5}$ who used method B. However, since they gave only values of $\Delta \omega$ for separate values of field strength, this requires fitting their data to a polynomial in $F$.

The change in intensity of the ground to fundamental transition for a diatomic molecule in the presence of an electric field is much less straightforward. The reason for this is that the intensity is proportional to the square of the integral $\left\langle\psi_{0}^{F}\left|\mu^{F}\right| \psi_{1}^{F}\right\rangle$ multiplied by $\omega^{F}$, where $\psi_{0}^{F}$ and $\psi_{1}^{F}$ are the field-perturbed ground and fundamental vibrational wave functions and $\mu^{F}$ is the dipole moment function in the presence of the field. The only way one can follow the spirit of method B is to approximate this integral as $\left\langle\psi_{0}\left|\mu^{F}\right| \psi_{1}\right\rangle$ which, in the harmonic oscillator approximation, is ( $\hbar /$ $\left.2 \omega_{e}\right)^{1 / 2}\left(\partial \mu^{F} / \partial Q\right)$, and to approximate $\omega^{F}$ by $\omega_{e}$. The derivative $\left(\partial \mu^{F} / \partial Q\right)$ can then be evaluated in terms of $F$ with the methodology of Sec. II B. Alternatively, applying the strategy of method A, the result reported in Ref. 4 is obtained, namely,

$$
\begin{aligned}
\left\langle\psi_{0}^{F}\left|\mu^{F}\right| \psi_{1}^{F}\right\rangle= & \left(\hbar / 2 \omega_{e}\right)^{1 / 2}\{(\partial \mu / \partial Q)+[(\partial \alpha / \partial Q) \\
& \left.+(5 / 4) \omega_{e}^{-2}(\partial \mu / \partial Q)\left(\partial^{2} \mu / \partial Q^{2}\right)\right] F \\
& \left.+[(\partial \beta / \partial Q)+\cdots] F^{2}\right\} .
\end{aligned}
$$

Comparison of the two methods shows agreement with the terms involving $(\partial \mu / \partial Q),(\partial \alpha / \partial Q)$, etc., but the factor $(5 / 4)$ in the second part of the term which is linear in $F$ is unity in method B; this is the result of ignoring the fielddependence of $\psi_{0}$ and $\psi_{1}$ in the initial transition integral. However, $\left|\left\langle\psi_{0}^{F}\left|\mu^{F}\right| \psi_{1}^{F}\right\rangle\right|^{2} \omega^{F}$ of method $\mathrm{A}$ and ( $\hbar /$ 2) $\left(\partial \mu^{F} / \partial Q\right)^{2}$ of method B do agree, and thus the intensities, up to the term linear in $F$ (when mechanical anharmonicity in $\omega^{F}$ is ignored), also agree and the (5/4) difference disappears. That is, in method $\mathrm{A}$ we find

$$
\begin{aligned}
\left|\left\langle\psi_{0}^{F}\left|\mu^{F}\right| \psi_{1}^{F}\right\rangle\right|^{2} \omega^{F}= & \left(\hbar / 2 \omega_{e}\right)\left\{(\partial \mu / \partial Q)^{2} \omega_{e}\right. \\
& +\left[2(\partial \mu / \partial Q)(\partial \alpha / \partial Q) \omega_{e}\right. \\
& +2(\partial \mu / \partial Q)^{2}\left(\partial^{2} \mu / \partial Q^{2}\right) \omega_{e}^{-1} \\
& \left.\left.+\frac{1}{2} k^{\prime}(\partial \mu / \partial Q)^{3} \omega_{e}^{-3}\right] F+\cdots\right\}
\end{aligned}
$$


and, in method $\mathrm{B}$, the expression for $(\hbar / 2)\left(\partial \mu^{F} / \partial Q\right)^{2}$ is the same except for the absence of the last term. The coefficient of $F^{2}$ in Eq. (35), except for $(\partial \beta / \partial Q)$, is quite different in the two approaches.

\section{CONCLUSIONS}

We have shown how two quite different approaches to the calculation of the effects of an electric field on molecular vibration can be interpreted and interconnected. The interpretation is relevant to both vibrational polarizabilities and hyperpolarizabilities and the vibrational Stark effect. In doing so we have developed new formulas for certain vibrational (hyper)polarizability components $\left(\left[\mu^{2}\right]^{0,2}\right.$ and $\left[\mu^{3}\right]^{n, m}$ for $n+m=3$ ) which were not given in Refs. 7 and 8 . The relations between the two methods have been found to be more complex than originally anticipated, but the results found should make comparison between calculations using the two methods more facile. It has also been seen that the development of method $B$ is simpler than that of method A, but a caveat must be added that method B cannot be easily extended to processes involving a dynamic electric field nor has its application to polyatomic molecules yet been clearly delineated.

\section{ACKNOWLEDGMENTS}

We thank Professor B. Kirtman for his friendly advice; D. M. B. thanks the Natural Sciences and Engineering Research Council of Canada and J. M. thanks the Generalitat de Catalunya for financial support.

${ }^{1}$ A. D. Buckingham, Adv. Chem. Phys. 12, 107 (1967).

${ }^{2}$ D. M. Bishop, Rev. Mod. Phys. 62, 343 (1990).

${ }^{3}$ C. E. Dykstra, J. Chem. Educ. 65, 198 (1988).

${ }^{4}$ D. M. Bishop, J. Chem. Phys. 98, 3179 (1993); there is a misprint in Eq. (3) of this article, the factor should read $1.7770 \times 10^{6}$ not 1.1770 $\times 10^{6}$. There is also a misprint in Eq. (10), the term after the summation sign should read $f_{a n n}(P / a) \omega_{a}^{-2}$. In this article, the frequencies given in a.u. are circular and defined by $E=\hbar \omega$, but all those in $\mathrm{cm}^{-1}$ are regular ones and defined by $E=h c \omega$.

${ }^{5}$ J. L. Andrés, M. Duran, A. Lledós, and J. Bertrán, Chem. Phys. 151, 37 (1991).

${ }^{6}$ B. Kirtman and D. M. Bishop, Chem. Phys. Lett. 175, 601 (1990).

${ }^{7}$ D. M. Bishop and B. Kirtman, J. Chem. Phys. 95, 2646 (1991).

${ }^{8}$ D. M. Bishop and B. Kirtman, J. Chem. Phys. 97, 5255 (1992).

${ }^{9}$ D. M. Bishop, B. Kirtman, H. A. Kurtz, and J. E. Rice, J. Chem. Phys. 98, 8024 (1993).

${ }^{10}$ D. M. Bishop and S. A. Solunac, Phys. Rev. Lett. 55, 1986, 2627 (1985).

${ }^{11}$ L. Adamowicz and R. J. Bartlett, J. Chem. Phys. 84, 4988 (1986); 86, 7250 (1987).

${ }^{12}$ J. Martí, J. L. Andrés, J. Bertrán, and M. Duran, Mol. Phys. (in press).

${ }^{13}$ J. Martí, A. Lledós, J. Bertrán, and M. Duran, J. Comput. Chem. 13, 821 (1992).

${ }^{14}$ E. B. Wilson, Jr., J. C. Decius, and P. C. Cross, Molecular Vibrations (McGraw-Hill, New York, 1955), Appendix III.

${ }^{15}$ D. K. Lambert, J. Chem. Phys. 94, 6237 (1991). 